\title{
Alpha Power Gates Relevant Information during Working Memory Updating
}

\author{
Peter Manza, Chui Luen Vera Hau, and Hoi-Chung Leung (梁海松) \\ Department of Psychology, Stony Brook University, Stony Brook, New York 11794
}

\begin{abstract}
Human working memory (WM) is inherently limited, so we must filter out irrelevant information in our environment or our mind while retaining limited important relevant contents. Previous work suggests that neural oscillations in the alpha band $(8-14 \mathrm{~Hz})$ play an important role in inhibiting incoming distracting information during attention and selective encoding tasks. However, whether alpha power is involved in inhibiting no-longer-relevant content or in representing relevant WM content is still debated. To clarify this issue, we manipulated the amount of relevant/irrelevant information using a task requiring spatial WM updating while measuring neural oscillatory activity via EEG and localized current sources across the scalp using a surface Laplacian transform. An initial memory set of two, four, or six spatial locations was to be memorized over a delay until an updating cue was presented indicating that only one or three locations remained relevant for a subsequent recognition test. Alpha amplitude varied with memory maintenance and updating demands among a cluster of left frontocentral electrodes. Greater postcue alpha power was associated with the high relevant load conditions (six and four dots cued to reduce to three relevant) relative to the lower load conditions (four and two dots reduced to one). Across subjects, this difference in alpha power was correlated with condition differences in performance accuracy. In contrast, no significant effects of irrelevant load were observed. These findings demonstrate that, during WM updating, alpha power reflects maintenance of relevant memory contents rather than suppression of no-longer-relevant memory traces.
\end{abstract}

Key words: alpha power; cognition; EEG; oscillations; updating; working memory

\section{Introduction}

Human spatial working memory (WM) capacity is estimated to be approximately five visual stimuli (Jiang et al., 2000). Therefore, the ability to maintain relevant information in WM while ignoring distractions is critical for healthy cognitive functioning. Computational models propose that neural oscillations aid in these processes (Lisman and Idiart, 1995). In particular, alphaband $(8-14 \mathrm{~Hz})$ activity has been implicated in supporting WM and other cognitive processes, although its exact function in cognition remains controversial.

A prevailing theory in the last decade suggests that alpha amplitude or power increases reflect a top-down mechanism of inhibition, controlling the timing of neuronal discharges in nearby populations (Klimesch et al., 2007). This inhibitory capacity of alpha-band activity is hypothesized as being the basic mechanism subserving various cognitive processes, including sensory stimulus processing and WM functions. Some strong evidence from

\footnotetext{
Received 0ct. 31, 2013; revised March 17, 2014; accepted March 23, 2014.

Author contributions: C.L.V.H. and H.-C.L. designed research; C.L.V.H. performed research; P.M. and C.L.V.H. analyzed data; P.M. and H.-C.L. wrote the paper.

This work was partially supported by the State University of New York at Stony Brook including the Office of the Vice President of Research. We thank undergraduate research assistants Chuck Vollmoeller, Ashu Kapoor, Holman Li, and Steven Marchessault for help with subject recruitment and data collection.

The authors declare no competing financial interests.

Correspondence should be addressed to Peter Manza or Hoi-Chung Leung, Department of Psychology, Stony Brook University, Stony Brook, NY 11794-2500, E-mail: peter.manza@stonybrook.edu or hoichung.leung@stonybrook.edu.

DOI:10.1523/JNEUROSCI.4641-13.2014

Copyright (C) 2014 the authors $\quad 0270-6474 / 14 / 345998-05 \$ 15.00 / 0$
}

studies of stimulus perception, attention, and selective WM encoding suggests that alpha power increases reflect sensory gating via disengagement of task-irrelevant sensory brain regions (for review, see Jensen and Mazaheri, 2010). However, the role of alpha activity in processing internal representations (e.g., WM maintenance and updating) has been heavily debated. Klimesch et al. (2007) proposed that delay-period increases in alpha during WM tasks are related to the suppression of previous memory representations that are no longer relevant. However, this hypothesis has only indirect supporting evidence (Klimesch et al., 1999) and has been contested by recent findings suggesting that alpha dynamics are directly related to relevant WM demands (Palva et al., 2011). One reason for this discrepancy is that WM tasks often require sensory processing that varies with the current WM demands, so the two are difficult to disentangle. Therefore, a direct manipulation of WM load demands independent of sensory stimuli is required.

To delineate the possible role of alpha power in WM updating and selective retrieval, we recorded electroencephalography (EEG) while subjects performed a visuospatial WM task with a retro cue inserted during the delay period, as others have done with behavioral research (Oberauer, 2001). This is in contrast to studies that used a precue manipulation to investigate perceptual/attention biases or selective WM encoding (Bonnefond and Jensen, 2012). The retro cue indicated that only a subset of the previously memorized information remained relevant for an upcoming recognition test. By systematically manipulating both postcue relevant and no-longer-relevant spatial WM load (with- 
a

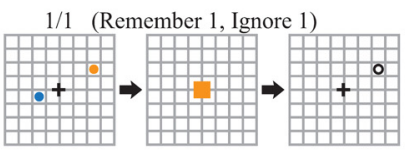

$1 / 3$ (Remember 1, Ignore 3)
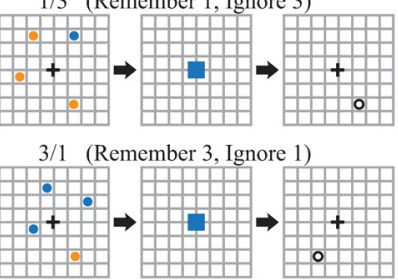

3/3 (Remember 3, Ignore 3)

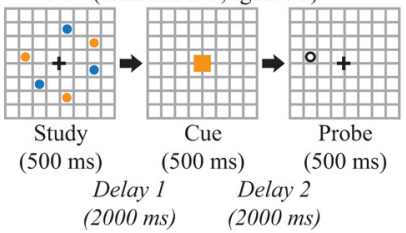

b

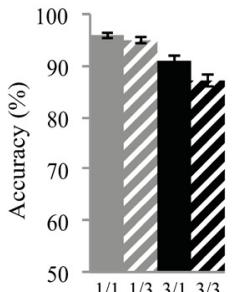

C

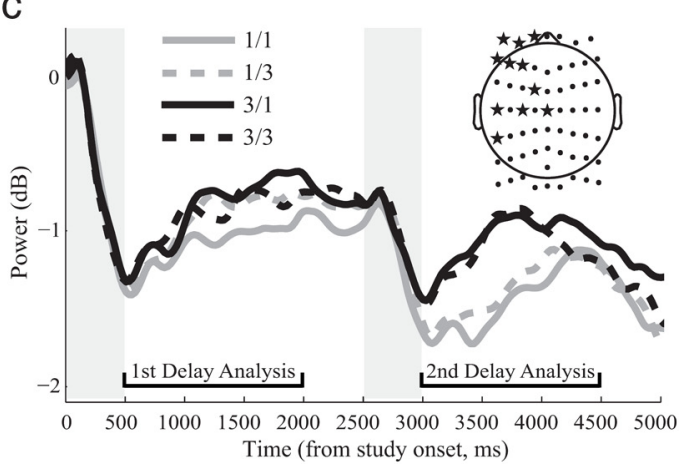

Figure 1. $\quad \boldsymbol{a}$, Spatial WM task with a retro cue. The initial memory set consisted of two, four, or six dots presented in different locations on each trial, followed by delay 1 (the study phase). An orange or blue updating cue then indicated the one ([1/1] and [1/3] conditions) or three ([3/1] and [3/3]) dot locations of the original memory set that remained relevant throughout delay 2 (the cue phase). Finally, a probe appeared prompting a recognition response. $\boldsymbol{b}$, Behavioral results indicating accuracy and RT differences between the four experimental conditions. c, Alpha power activity for the full trial of all four conditions. Gray shaded regions indicate the study and cue displays. Top right inset, Montage of scalp electrodes. Starred electrodes are significant at the $p<0.05$ level over the delay 2 period (500-2000 ms postcue onset; see Materials and Methods).

out varying sensory demands during updating), we tested relevant/irrelevant WM load-dependent changes in alpha power directly. We found that cue-related alpha increases were dependent on relevant WM load, localized to frontocentral regions implicated in WM processing, and related to condition differences in behavioral accuracy. These results suggest that alpha is not necessarily related to the inhibition of previous WM traces and provide a different framework for interpreting alpha's function in WM.

\section{Materials and Methods}

Data. The data used in the present study were taken from a previous experiment that examined event-related potentials (Hau, 2012). Here, we reanalyzed the data to examine oscillatory neural activity during spatial WM updating. Full details of the task design and procedures may be found in Hau (2012).

Participants. Thirty-six healthy individuals (20 female; mean age $=$ 19.72 years) participated. All participants had normal or corrected-tonormal vision and self-reported having no history of neurological disorder, psychiatric disorder, or drug abuse. The protocol was approved by the local Institutional Review Board. Four datasets were excluded for EEG problems. In addition, one dataset was excluded for performance 3 SDs away from the mean. As a result, data from 31 subjects ( 16 female; mean age $=19.50$ years) were included in the final analysis.

Spatial WM task with an updating cue. Subjects performed a delayedrecognition task with four conditions in which an updating cue (also called a "retro cue") was inserted during the delay (task adapted and modified from Leung and Zhang, 2004; Fig. 1a). Trials began with a $0.3 \mathrm{~s}$ fixation period followed by a $0.5 \mathrm{~s}$ stimulus display of two, four, or six dots (one or three in blue and one or three in orange) in different grid locations. These dot locations comprised the initial memory set and were to be remembered over a $2 \mathrm{~s}$ delay until cue presentation $(0.5 \mathrm{~s})$. The cue was either a blue or orange square, indicating that only the locations of dots in the cued color remained relevant for an upcoming recognition test. Therefore, the final postcue memory set size was reduced to one or three dot locations. All cues were $100 \%$ valid. After another $2 \mathrm{~s}$ delay, a probe $(0.5 \mathrm{~s})$ was presented instructing participants to make a button press to indicate whether it matched one of the dot locations in the final memory set as quickly and accurately as possible. There were two probe types (50/50 chance): (1) match (yes), the probe matched a relevant dot location, or (2) nonmatch (no), the probe did not match a relevant dot location. To reduce trial history effects, the probe of the current trial never appeared in the preceding two trials. Each study and probe location combination was unique (no repeat trials) and was presented pseudorandomly. Cue color and probe types were also pseudorandomly selected and counterbalanced across runs. Intertrial intervals were $1.5 \mathrm{~s}$ each, resulting in trials that were $7.3 \mathrm{~s}$ long. The experiment consisted of 512 experimental and eight catch trials divided into eight runs. Each run included a catch trial at the start and four blocks of 16 experimental trials. A $10 \mathrm{~s}$ resting break was given between blocks. Subjects practiced for one to three blocks (20 trials each) before beginning.

The four task conditions were named to reflect the number of relevant and irrelevant items in the final memory set after cue presentation: (a) $1 / 1$, (b) $1 / 3$, (c) $3 / 1$, and (d) $3 / 3$. The sum of the two numbers is the initial memory load before cue presentation. For examples, 1/1 indicates that two dots at the study display were reduced to one relevant dot location after the cue with one dot rendered irrelevant; $1 / 3$ indicates that four dots at the study display were reduced to one relevant dot location after the cue with three dots rendered irrelevant, and so forth.

EEG recording and data analysis. EEG was continuously recorded from $64 \mathrm{Ag} / \mathrm{AgCl}$ electrodes in a cap (Neuroscan) according to the International 10-20 system, with a frontocentral electrode as the ground and a linked-mastoid reference (for details, see Hau, 2012). Electrode impedance was kept $<2 \mathrm{k} \Omega$. EEG data were recorded at $1000 \mathrm{~Hz}$ and amplified with a DC to $30 \mathrm{~Hz}$ band-pass filter.

All EEG preprocessing and analyses were conducted using the EEGLab toolbox (version 12, www.sccn.ucsd.edu/eeglab/; Delorme and Makeig, 2004). The EEG data were downsampled to $250 \mathrm{~Hz}$ and band-pass filtered from 1 to $30 \mathrm{~Hz}$. Epochs were extracted (from correct trials only) from $800 \mathrm{~ms}$ prestimulus onset to $1500 \mathrm{~ms}$ postprobe onset, resulting in 7300 ms epochs. Trials with drifting or large movement artifacts were removed by visual inspection before analysis. Independent component analysis was applied to the data to remove the effects of blinks, eye movements, and muscle/cardiac artifacts. Remaining trials with EEG voltages exceeding $100 \mu \mathrm{V}$ measured from peak-to-peak at any channel were also removed using ERPLab (www.erpinfo.org/erplab). After all preprocessing, on average, 388 trials $(76 \%)$ remained per subject (range $=297-465$; $\mathrm{SD}=37.69$ )

After preprocessing, we applied a scalp surface Laplacian transformation to the EEG data using the Current Source Density toolbox (version 1.1; Kayser and Tenke, 2006). We used a spherical spline surface Laplacian measure (Perrin et al., 1989) with $m$-constant $=4$ and smoothing constant $\lambda=10^{-6}$. Spectral power was then computed by convolving 6-cycle complex Morlet wavelets with each single-trial EEG epoch for each electrode from 6 to $30 \mathrm{~Hz}$ in $1 \mathrm{~Hz}$ steps. The $250 \mathrm{~ms}$ preceding the study stimulus onset was used as a baseline. The magnitude of the resulting complex numbers was squared to give an estimate of power at each frequency (Roach and Mathalon, 2008). For statistical analyses, we analyzed the 500-2000 ms time windows during both the precue and postcue delays, omitting the first and last $500 \mathrm{~ms}$ of each phase (Fig. 1c). This was done to avoid polluting the oscillatory estimates with activity from other portions of the trial because wavelet analyses lack temporal preci- 
sion. We then averaged wavelets with center frequencies in the alpha $(8-14 \mathrm{~Hz})$ range together. Exploratory analyses of other frequencies yielded no significant results.

To find the electrodes of interest for statistical analyses, we used a permutation-based clustering procedure (Maris and Oostenveld, 2007). This allowed us to determine, without bias, the sensors where significant alpha power differences exist between our four experimental conditions while avoiding the multiple-comparisons problem. The clustering procedure was performed in the $8-14 \mathrm{~Hz}$ range over the postcue delay period (500-2000 ms postcue onset) with a $p<0.05$ threshold and 500 random permutations of the data. Because alpha oscillations have been shown to be involved in a wide range of cognitive functions in various cortical regions (Başar, 2012), we chose this time period for electrode selection to study WM updating specifically. This way of electrode selection is comparable to other studies that have used the equivalent of our first delay to study WM maintenance. Because all four conditions were used in this procedure, the electrodes were not selected for any specific hypothesis or for isolating any particular effects (such as relevant or irrelevant load effects).

Finally, all electrodes identified as significant via the clustering procedure were averaged together and used for further statistical analyses. In particular, we performed ANOVAs to test for condition differences in alpha power during the study and cue delays. For each ANOVA in which the assumption of sphericity was violated, a Greenhouse-Geisser correction was used to adjust for degrees of freedom.

\section{Results}

\section{Behavioral performance}

ANOVAs across the four conditions revealed significant differences among the four conditions in average accuracy and response time to the probes $\left(F_{(2.19,65.72)}=51.38, p<0.001\right.$, and $F_{(1.53,45.96)}=151.70, p<0.001$, respectively; Figure $\left.1 b\right)$. Paired $t$ tests further revealed that both relevant load effects $[$ mean $(3 / 3,3 / 1)-\operatorname{mean}(1 / 3,1 / 1)]$ and irrelevant load effects $[$ mean $(3 / 3,1 / 3)-$ mean $(3 / 1,1 / 1)]$ were significant for accuracy and response time $(p<0.01)$, with less accurate and slower responses on trials of higher relevant and irrelevant load relative to trials of lower load $(p<0.01)$. Therefore, both higher relevant and irrelevant loads significantly affected recognition performance.

\section{Spectral alpha power}

We conducted the cluster correction procedure (see Materials and Methods) using data from the postcue delay. This analysis yielded 11 electrodes in the frontal and central regions with significant differences among the four experimental conditions at the $p<0.05$ level (Fig. $1 c$, inset). All of the following analyses refer to the averages of these 11 electrodes.

Overall, alpha power values during the two delay periods were significantly suppressed from baseline values for all four conditions $(t>5.90, p<0.001)$, as in previous studies (Palva et al., 2011). Nevertheless, alpha power was modulated by task condition in the precue and postcue phases of the task (Fig. 1c).

\section{Effect of load on study phase alpha activity}

To replicate previous experiments showing WM-load-dependent changes in alpha power (Jensen et al., 2002), we examined alpha activity during the initial delay (500-2000 ms poststimulus). Alpha power differences were approaching significance for the four conditions $\left(F_{(3,90)}=2.07, p=0.10\right)$. This effect was driven mostly by the 2 -item set size ( $1 / 1$ condition), which had marginally lower power than the 4- and 6-item set sizes over this time $(p<0.10$, post hoc $t$ tests). The six-location condition was not significantly higher than the four-location conditions $(p>0.65)$, probably because six locations exceeded the average spatial WM capacity limit. Previous studies have shown that neural activity plateaus around four items in visuospatial WM (Leung et al., 2004). Condition differences in alpha power during the study phase were not significantly related to behavioral performance.

Because the cluster of electrodes were derived from the second delay (see Materials and Methods), we conducted an additional clustering analysis using data from this first delay. This was to ensure that any alpha effect during the first delay was not missed due to the electrode selection procedure. No clusters were significant at the $p<0.05$ level. However, at a less stringent level $(p<$ 0.09 ), we found a cluster of several posterior electrodes that showed a similar pattern of load effect during this first delay. This cluster was not used in any further analysis because it was not above threshold.

\section{Effect of relevant versus irrelevant load on postcue alpha activity}

Alpha power for the 500-2000 ms period postcue onset was significantly modulated by condition $\left(F_{(3,90)}=5.65, p=0.001\right)$. Post hoc $t$ tests revealed that the conditions were separated by relevant load, such that $3 / 1$ and $3 / 3$ had significantly higher alpha power than $1 / 1$ and $1 / 3$, respectively $(p<0.05)$. In contrast, there were no significant effects of irrelevant load, such that $1 / 3$ was not significantly different from $1 / 1$ and $3 / 3$ was not significantly different from $3 / 1(p>0.50)$. Similar effects were observed when averaging conditions to examine the relevant load effects $(p<$ 0.001 ; Fig. $2 a$, top) and irrelevant load effects ( $p>0.50$; Fig. $2 a$, bottom). To assess the reliability of this relevant load effect, we conducted a split-half reliability test. We analyzed the oddnumbered trials and the even-numbered trials separately and then tested whether the differences in alpha power (high relevant loads - low relevant loads) from the two analyses were correlated among all 31 subjects. We found that they were highly correlated $(r=0.766$, Spearman-Brown coefficient $=0.868)$, suggesting that the effect was consistent across the different trials of the experiment. It should be noted that whereas the selected cluster of 11 frontocentral electrodes show a significant and reliable relevant load effect, the topography suggests that this effect may be present throughout much of the scalp (Fig. $2 a$, top, inset).

To determine whether we missed any significant irrelevant load effects during the postcue phase due to the electrode selection procedure, we conducted another clustering analysis during the postcue delay and specifically assessed the irrelevant load effect. This time, rather than using all four conditions, we used the combined high irrelevant loads (1/3 and 3/3) and compared that with the combined low irrelevant loads (1/1 and 3/1). This analysis yielded no significant clusters, even at a reduced threshold of $p<0.15$ (see topography; Fig. $2 a$, bottom inset).

To further evaluate alpha's role in WM updating, we examined the relationship between alpha power and behavioral performance for the relevant and irrelevant load effects. Across subjects, the difference between high relevant versus low relevant alpha power during the postcue delay (500-2000 ms) showed a moderate negative correlation with the response accuracy difference between them $(r=-0.45, p<0.01$; Fig. $2 b$, top). In a follow-up median split analysis, subjects with lower relevant load differences in alpha power $(n=15)$ performed significantly worse at high relevant loads $\left(t_{(29)}=-2.12, p<0.05\right)$, with a $2.7 \%$ drop in accuracy relative to low relevant loads. This difference in alpha power, however, did not correlate with overall performance accuracy or reaction time (RT; $p>0.10)$. There were also no significant correlations in overall accuracy, RT, or condition differences in accuracy and RT for irrelevant load alpha power differences $(p>0.10)$. 
a
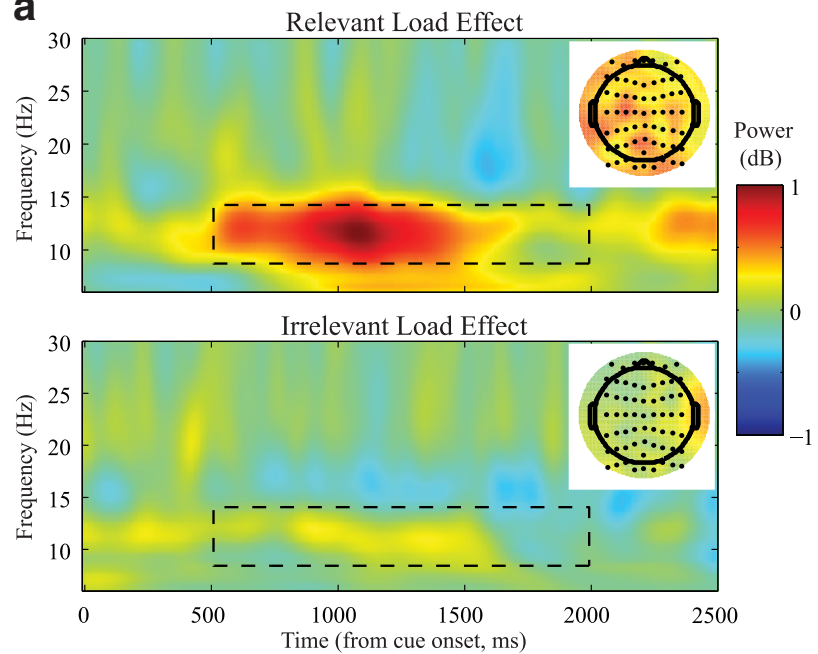

b

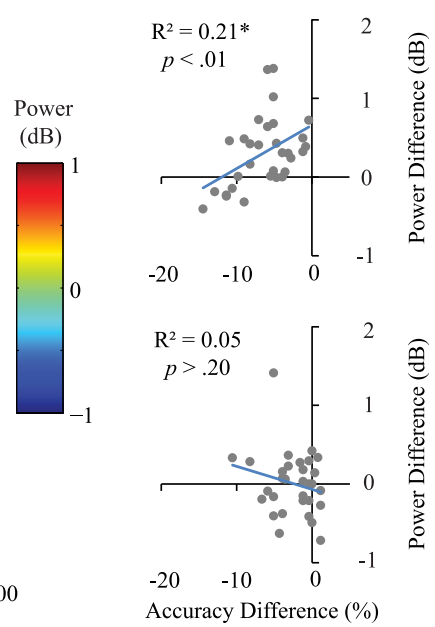

Figure 2. $\quad \boldsymbol{a}$, Effects of relevant/irrelevant memory load on postcue alpha power. Top, Relevant load effect, or the mean of 3/3, $3 / 1$ minus the mean of $1 / 3,1 / 1$. Bottom, Irrelevant load effect, or the mean of $3 / 3,1 / 3$ minus the mean of $3 / 1,1 / 1$. Dashed lines indicate the boundaries of the time-frequency window used for analysis. Spectral estimates shown are smoothed linearly in both the time and frequency dimension for better visualization. Insets, Topography for the relevant (top) and irrelevant (bottom) load effects. $\boldsymbol{b}$, Task performance was associated with relevant WM load alpha effect after cued updating. Top, Correlation between the relevant load effect for alpha power difference and the relevant load effect for behavioral accuracy across subjects. Bottom, Correlation between the irrelevant load effect for alpha power difference and the irrelevant load effect for behavioral accuracy across subjects.

\section{Discussion}

Using a spatial WM paradigm with a retro-cue manipulation, we demonstrate here that postcue alpha-band $(8-14 \mathrm{~Hz})$ activity in frontocentral regions is modulated by the relevant memory load demand rather than by the irrelevant load demand. We also found that these relevant load-related changes in alpha were related to behavioral accuracy. These findings suggest that, when selectively retrieving spatial information in WM, frontocentral alpha oscillations are directly related to the relevant memory demand, but may not be related to the inhibition of no-longerrelevant memory traces. In the following paragraphs we discuss the potential specific and general roles of alpha oscillations in WM.

We replicated some previous findings showing a general depression in alpha power relative to baseline (Palva et al., 2011) and load-dependent increases during WM maintenance (Jensen et al., 2002; Tuladhar et al., 2007; Palva et al., 2011; Roux et al., 2012; although note that the first two studies found these increases over posterior electrodes). The retro-cue manipulation allowed us to examine the effects of spatial WM updating beyond visual perceptual/attentional effects (which could be an issue if a precue manipulation was used) and to assess the dominant hypothesis that alpha power increases suppress task-irrelevant memory (Klimesch et al., 2007). We predicted that if increases in alpha power reflected successful distractor inhibition, we would observe an irrelevant load effect on postcue alpha activity. Instead, we observed a significant effect of postcue relevant memory load on alpha power estimates and insignificant effects of irrelevant memory load.

Further, the relevant load difference (but not the irrelevant load difference) in alpha was related to performance; subjects showing less of an alpha increase at higher loads had a greater drop in performance accuracy at higher loads. This suggests that the increases in alpha activity are a behaviorally adaptive mechanism to meet the demands of increasing WM load. Individuals

who do not show strong alpha increases are more likely to have performance suffer when WM demands are high. Note that we did not see correlations of this postcue alpha difference with the overall performance accuracy on the task as others did using a precue design (Palva et al., 2011). Therefore, the difference in alpha we observed is probably not a signature of strong overall performance or of those with a high WM capacity. We believe that this pattern may reflect the successful gating of task-relevant contents (see next paragraph) in WM to avoid the loss of performance typically observed as WM load increases.

Neural oscillations in the alpha range probably play a role in WM that is not solely related to either relevant or irrelevant WM demands. Rather, we propose that alpha's function varies depending on the context and may be related to the "gating" function of WM described by Hazy et al. (2006). This computational theory holds that basal ganglia-prefrontal interactions gate two competing WM processes: robust maintenance and dynamic updating. When WM contents need to be updated, or when WM demands are not high, the "gate" opens to allow for processing of incoming information. A "closed gate" could be associated with increases in alpha power, whereas an "open gate" is related to decreases.

This interpretation provides a useful distinction to explain the different findings among previous studies and the present study. Earlier experiments examining selective encoding of external stimuli using a precue design found that, during WM encoding and maintenance, posterior alpha power increased with the presence of additional irrelevant stimuli (Bonnefond and Jensen, 2012). In this context, increases in posterior alpha power may reflect a closed gate in the sensory-related brain regions, protecting the perception and encoding of relevant stimuli against intruding sensory stimulation. In the present study, which had a retro-cue design, the task was to selectively retrieve relevant information from existing WM and we found that frontocentral alpha power increased with relevant load only (i.e., a different topography compared with previous studies of sensory gating). Here, increases in alpha power may also reflect a closed gate, but in this context, it is to protect internal WM representations from a switch to updating processes (at the expense of maintenance). Alternatively, one could view these findings from the opposite standpoint: in the lower relevant load conditions (1/1 and 1/3), more resources are available to update and process incoming information, so alpha power decreases in these conditions may reflect an open gate. Critically, sensory stimulus load did not vary at the updating stage in our paradigm. Therefore, posterior alpha power did not increase with irrelevant WM load because there was no need to block off distracting sensory information.

Although it is also possible that our discrepant results may be simply due to the frontocentral scalp location of the effects, we observed little or no irrelevant load effects anywhere on the scalp, even at low thresholds. Alpha power modulations have been reported in almost every major cortical region (Başar, 2012). Some have further suggested that alpha power modulations serve dif- 
ferent functions depending on cortical location (Mo et al., 2011), and generators of the alpha rhythm exist in different cortical layers depending on the specific region where they are located (Bollimunta et al., 2011). The frontal alpha activity observed in the present study might therefore have some functional differences compared with the posterior alpha activity found previously (e.g., memory gating vs sensory gating, respectively).

In sum, frontocentral alpha oscillations may have a role in gating WM or manipulating internal representations independently of sensory stimulation. Frontal alpha increases have been observed previously when comparing mentally rotating a visuospatial array to only retaining the array in WM (Sauseng et al., 2005). Future research should study directly whether the frontal alpha effects seen here are specific to spatial WM updating and whether the spatiotemporal pattern of alpha power depends on external versus internal information filtering. Whereas our present data can only be applied to spatial WM updating, we propose that the gating function of alpha-band activity would apply in many other contexts.

\section{References}

Başar E (2012) A review of alpha activity in integrative brain function: Fundamental physiology, sensory coding, cognition and pathology. Int J Psychophysiol 86:1-24. CrossRef Medline

Bollimunta A, Mo J, Schroeder CE, Ding M (2011) Neuronal mechanisms and attentional modulation of corticothalamic $\alpha$ oscillations. J Neurosci 31:4935-4943. CrossRef Medline

Bonnefond M, Jensen O (2012) Alpha oscillations serve to protect working memory maintenance against anticipated distracters. Curr Biol 22:19691974. CrossRef Medline

Delorme A, Makeig S (2004) EEGLAB: an open source toolbox for analysis of single-trial EEG dynamics including independent component analysis. J Neurosci Methods 134:9-21. CrossRef Medline

Hau CLV (2012) Event-related potential (ERP) studies of spatial working memory. Doctoral dissertation. Stony Brook University, Stony Brook, NY.

Hazy TE, Frank MJ, O’Reilly RC (2006) Banishing the homunculus: making working memory work. Neuroscience 139:105-118. CrossRef Medline

Jensen O, Mazaheri A (2010) Shaping functional architecture by oscillatory alpha activity: gating by inhibition. Front Hum Neurosci 4:186. CrossRef Medline

Jensen O, Gelfand J, Kounios J, Lisman JE (2002) Oscillations in the alpha band $(9-12 \mathrm{~Hz})$ increase with memory load during retention in a shortterm memory task. Cereb Cortex 12:877-882. CrossRef Medline
Jiang Y, Olson IR, Chun MM (2000) Organization of visual short-term memory. J Exp Psychol Learn Mem Cogn 26:683-702. CrossRef Medline

Kayser J, Tenke CE (2006) Principal components analysis of Laplacian waveforms as a generic method for identifying ERP generator patterns. I. Evaluation with auditory oddball tasks. Clin Neurophysiol 117:348-368. CrossRef Medline

Klimesch W, Doppelmayr M, Schwaiger J, Auinger P, Winkler T (1999) "Paradoxical" alpha synchronization in a memory task. Brain Res Cogn Brain Res 7:493-501. CrossRef Medline

Klimesch W, Sauseng P, Hanslmayr S (2007) EEG alpha oscillations: the inhibition-timing hypothesis. Brain Res Rev 53:63-88. CrossRef Medline

Leung HC, Zhang JX (2004) Interference resolution in spatial working memory. Neuroimage 23:1013-1019. CrossRef Medline

Leung HC, Seelig D, Gore JC (2004) The effect of memory load on cortical activity in the spatial working memory circuit. Cogn Affect Behav Neurosci 4:553-563. CrossRef Medline

Lisman JE, Idiart MA (1995) Storage of 7+/-2 short-term memories in oscillatory subcycles. Science 267:1512-1515. CrossRef Medline

Maris E, Oostenveld R (2007) Nonparametric statistical testing of EEG- and MEG-data. J Neurosci Methods 164:177-190. CrossRef Medline

Mo J, Schroeder CE, Ding M (2011) Attentional modulation of alpha oscillations in macaque inferotemporal cortex. J Neurosci 31:878-882. CrossRef Medline

Oberauer K (2001) Removing irrelevant information from working memory: a cognitive aging study with the modified Sternberg task. J Exp Psychol Learn Mem Cogn 27:948-957. CrossRef Medline

Palva S, Kulashekhar S, Hämäläinen M, Palva JM (2011) Localization of cortical phase and amplitude dynamics during visual working memory encoding and retention. J Neurosci 31:5013-5025. CrossRef Medline

Perrin F, Pernier J, Bertrand O, Echallier JF (1989) Spherical splines for scalp potential and current density mapping. Electroencephalogr Clin Neurophysiol 72:184-187. CrossRef Medline

Roach BJ, Mathalon DH (2008) Event-related EEG time-frequency analysis: an overview of measures and an analysis of early gamma band phase locking in schizophrenia. Schizophr Bull 34:907-926. CrossRef Medline

Roux F, Wibral M, Mohr HM, Singer W, Uhlhaas PJ (2012) Gamma-band activity in human prefrontal cortex codes for the number of relevant items maintained in working memory. J Neurosci 32:12411-12420. CrossRef Medline

Sauseng P, Klimesch W, Doppelmayr M, Pecherstorfer T, Freunberger R, Hanslmayr S (2005) EEG alpha synchronization and functional coupling during top-down processing in a working memory task. Hum Brain Mapp 26:148-155. CrossRef Medline

Tuladhar AM, ter Huurne N, Schoffelen JM, Maris E, Oostenveld R, Jensen O (2007) Parieto-occipital sources account for the increase in alpha activity with working memory load. Hum Brain Mapp 28:785-792. CrossRef Medline 